\title{
Efetividade da Musicoterapia na Redução da Ansiedade de Pacientes Oncológicos: Revisão Sistemática
}

doi: https://doi.org/10.32635/2176-9745.RBC.2019v65n4.592

\author{
Effectiveness of Music Therapy in Reducing Anxiety in Cancer Patients: Systematic Review \\ Efectividad de la Musicoterapia en la Reducción de la Ansiedad en Pacientes con Cáncer: Revisión Sistemática
}

\author{
Carolina Barbosa Neres'; Keyla de Paula Barbosa²; Patrícia Azevedo Garcia ${ }^{3}$; Aline Teixeira Alves; Liana Barbaresco Gomide \\ Matheus $^{5}$
}

Resumo

Introduçáo: $\mathrm{O}$ câncer é uma doença que envolve extenso sofrimento emocional, físico e social, o que favorece o aparecimento de diversas morbidades, incluindo ansiedade. Terapias complementares, como a musicoterapia, têm sido estudadas como alternativas para a abordagem da ansiedade. Objetivo: Revisar sistematicamente os estudos e determinar a efetividade da musicoterapia na redução da ansiedade de pacientes oncológicos. Método: A pesquisa foi realizada em seis bases de dados incluindo MEDLINE/PubMed, SciELO, Scopus, CINAHL, Cochrane e Web of Science, sem restrição de data, sexo, etnia ou tipos de câncer. Foram incluídos somente ensaios clínicos randomizados que avaliaram a ansiedade como um dos desfechos, bem como os que utilizaram a musicoterapia como intervenção e que envolveram amostra com indivíduos adultos diagnosticados com câncer. Foram excluídos os estudos em que a musicoterapia não foi realizada por um profissional especializado, não foi proposto grupo controle, não foi disponibilizado o estudo na integra, associaram pacientes com outras doenças além do câncer e incluíram no grupo experimental outra intervenção além da música. Resultado: Foram encontrados 1.909 estudos, sendo oito elegíveis. A maioria dos estudos demonstrou benefícios da musicoterapia na ansiedade. Conclusáo: A musicoterapia é efetiva na reduçáo da ansiedade de pessoas com câncer. No entanto, mais estudos com novas tecnologias e mais detalhes sobre a intervenção são necessários para a confirmação dos resultados.

Palavras-chave: Musicoterapia; Ansiedade/terapia; Neoplasias.

\begin{abstract}
Introduction: Cancer is a disease that involves extensive emotional, physical and social suffering, which favors the appearance of various morbidities, including anxiety. Complementary therapies, such as music therapy, have been studied as alternatives to an approach to anxiety. Objective: To conduct a systematic review of the literature and determine the effectiveness of music therapy to reduce anxiety of oncologic patients. Method: The research was conducted in six databases including MEDLINE/PubMed, SciELO, Scopus, CINAHL, Cochrane and Web of Science without restriction of date, gender, ethnicity or cancer types. It were included only randomized controlled trials that evaluated anxiety as one of the outcomes, as well as those using music therapy as an intervention, involving a sample with adult individuals diagnosed with cancer. The exclusion criteria were studies where music therapy was not performed by a skilled professional, without control group, studies not fully available, that associated patients with diseases other than cancer and which included in the experimental group another intervention instead of music. Result: A total of 1909 studies were found, of which eight were eligible. The majority of the studies have demonstrated benefits of music therapy for anxiety. Conclusion: Music therapy is effective in reducing the anxiety of people with cancer. However, more studies with new methodologies and further details about the intervention are necessary to confirm the results.

Key words: Music Therapy; Anxiety/therapy; Neoplasms.
\end{abstract}

Resumen

Introducción: El cáncer es una enfermedad que implica extensión emocional, física y social, lo que favorece la aparición de diversas morbilidades, incluida la ansiedad. Las terapias complementarias, como la musicoterapia, se han estudiado como alternativas al enfoque de la ansiedad. Objetivo: Revisar la literatura sobre la efectividad de la musicoterapia para el tratamiento de pacientes con trastornos de ansiedad y cáncer. Método: La investigación se realizó en seis bases de datos, incluyendo MEDLINE/ PubMed, SciELO, Scopus, CINAHL, Cochrane y Web of Science, sin restricciones de fecha, género, etnia o tipos de cáncer. Se incluyeron solo ensayos controlados aleatorios de estudios que evaluaron la ansiedad como uno de los resultados, así como el uso de la musicoterapia como una intervención y la participación de una muestra con individuos adultos diagnosticados con cáncer. Los estudios en los que la musicoterapia no fue realizada por un profesional especializado, no se propuso un grupo de control, no se disponía de un estudio completo, se asociaron pacientes con enfermedades distintas al cáncer y se incluyó otra intervención además de la música en el grupo experimental. Resultados: Se encontraron 1.909 estudios, de los cuales ocho fueron elegibles. La mayoría de los estudios han demostrado los beneficios de la musicoterapia en la ansiedad. Conclusión: Esta revisión sistemática concluyó que la musicoterapia es efectiva para reducir la ansiedad de las personas con cáncer. Sin embargo, se necesitan más estudios con nuevas tecnologías y más detalles sobre la intervención para confirmar los resultados.

Palabras clave: Musicoterapia; Ansiedad/terapia; Neoplasias.

\footnotetext{
${ }^{1}$ Hospital Universitário de Brasília. Brasília (DF), Brasil. Orcid iD: https://orcid.org/0000-0002-5488-4476

${ }^{2}$ Programa de Pós-Graduação em Ciências da Reabilitação (PPG-CR)/Universidade de Brasília (UnB). Brasília (DF), Brasil. Orcid iD: https://orcid.org/0000-0002-6858-7237

${ }^{3}$ PPG-CR/UnB. Brasília (DF), Brasil. Orcid iD: https://orcid.org/0000-0002-9043-1386

${ }^{4}$ PPG-CR/UnB. Brasília (DF), Brasil. Orcid iD: https://orcid.org/0000-0003-0262-7475

${ }^{5}$ PPG-CR/UnB. Brasília (DF), Brasil. Orcid iD: https://orcid.org/0000-0002-2574-9697

Endereço para correspondência: Keyla de Paula Barbosa. SHIN, CA 05, LTL1, apto. 07 - Lago Norte. Brasília (DF), Brasil. CEP 71503-505. E-mail: keylapaulab@gmail.com
} 


\section{INTRODUÇÃO}

O câncer é uma doença que envolve extenso sofrimento emocional, físico e social ${ }^{1}$. A visão pessimista do prognóstico, a vulnerabilidade do paciente e a possibilidade de recidiva favorecem o aparecimento de diversas morbidades, incluindo ansiedade ${ }^{2,3}$. A ansiedade é considerada uma antecipação de ameaça futura, caracterizada por sentimentos de apreensão, tensão, incapacidade de relaxar e comportamentos de cautela e esquiva. Essa desordem pode influenciar negativamente no enfrentamento da doença e na adesão necessária ao tratamento, como, por exemplo, na adesão à medicaçáo e nas idas às sessôes de radioterapia ${ }^{4}$, podendo se tornar crônica, influenciar negativamente nos efeitos colaterais do tratamento ${ }^{4}$ e interferir na vida, predispondo a outras doenças ${ }^{5}$. Nos últimos anos, terapias complementares, como a musicoterapia, têm sido estudadas como alternativas para a abordagem da ansiedade ${ }^{6}$.

A Associação Americana de Musicoterapia definiu a musicoterapia como uma prática baseada em evidências na qual musicoterapeutas certificados utilizam a música com objetivos terapêuticos específicos ${ }^{6}$ e metas individualizadas ${ }^{7}$. Diversas técnicas podem ser utilizadas a depender das necessidades do paciente, preferências e avaliação do musicoterapeuta ${ }^{8}$. O objetivo é que a música provoque um efeito distrator, focando a atenção do paciente longe de estímulos negativos para algo agradável e encorajador ${ }^{9}$. Alguns estudos investigaram o efeito da musicoterapia na ansiedade, no entanto, apresentaram resultados conflitantes ${ }^{10-14}$. Não foi encontrada nenhuma revisão sistemática que abordasse especificamente o efeito da musicoterapia na ansiedade do paciente oncológico. Nesse sentido, o objetivo do presente estudo é revisar sistematicamente os estudos e determinar a efetividade da musicoterapia na redução da ansiedade de pacientes oncológicos.

\section{MÉTODO}

Esta revisão incluiu ensaios clínicos randomizados de pacientes com câncer que realizaram musicoterapia para tratamento de ansiedade. O estudo seguiu as recomendaçóes do Preferred Reporting Items for Systematic Reviews and Meta-Analysis (PRISMA) e possui registro no PROSPERO (CRD42019119636).

A pesquisa foi realizada em seis bases de dados incluindo MEDLINE/PubMed, SciELO, Scopus, CINAHL, Cochrane e Web of Science, sem restrição de data, sexo, etnia ou tipos de câncer. A estratégia de busca utilizou os descritores obtidos no Medical Subject Headings (MESH) of the National Library of Medicine com a combinação de palavras-chave e os seguintes operadores boleanos ("Therapy, music" OR "music" OR "music therapy" $O R$ "music intervention" $O R$ "complementary therapies" $O R$ "alternative therapies" OR "integrative therapies") $A N D$ ("anxiety" OR "social anxiety") AND ("clinical trial" OR "randomized controlled trial" $O R$ "intervention study" $O R$ "trial") AND ("Neoplasms" OR "Neoplasm" OR "neoplasia" $O R$ "neoplasias" $O R$ "tumors" $O R$ "tumor" $O R$ "Cancer" $O R$ "Cancers" $O R$ "Malignant" $O R$ "Malignancy" $O R$ "Malignancies" OR "Carcinoma").

Foram incluídos somente ensaios clínicos randomizados, conforme recomendação da Cochrane Collaboration Handbook de estudos que avaliaram a ansiedade como um dos desfechos, bem como que utilizaram a musicoterapia como intervenção e que envolveram amostra com indivíduos adultos diagnosticados com câncer. Foram excluídos os estudos em que a musicoterapia não foi realizada por um profissional especializado, não foi proposto grupo controle, não foi disponibilizado o estudo na integra, associaram pacientes com outras doenças além do câncer e incluíram no mesmo grupo experimental outra intervenção além da música.

Os artigos foram selecionados por duas autoras após uma leitura sequenciada do título, resumo e texto completo, sempre nessa ordem. A lista de referências dos artigos foi consultada para encontrar possíveis estudos adicionais. Foram removidos os estudos duplicados. Os seguintes dados foram extraídos e inseridos em um formulário padronizado no Excel: autor, data, título, amostra, tipo de câncer, objetivo do estudo, tamanho da amostra (n), idade (anos), grupos, duração (semanas), frequência, intervenção, medidas de desfecho e conclusão do autor. Não foi utilizado filtro para data, tipo de estudo ou idiomas.

A qualidade dos estudos foi classificada por dois pesquisadores independentes que realizaram a avaliação por meio da Escala PEDro e do risco de viés (Cochrane) para todos os estudos incluídos nesta revisão. Todos os autores foram cegados para as avaliaçōes e as divergências foram resolvidas por um terceiro pesquisador.

\section{RESULTADOS}

Foram encontrados 1.909 estudos, sendo 1.493 após eliminação dos duplicados. Depois da leitura do título e resumo, permaneceram 47 artigos que foram lidos na íntegra. Destes, 39 foram eliminados pelos seguintes motivos: 14 estudos $^{15-28}$ não estavam disponíveis na integra; em $\mathrm{dez}^{29-38}$, a intervenção não foi realizada por um profissional musicoterapeuta; sete ${ }^{39-45}$ não tinham grupo controle; em seis estudos ${ }^{46-51}$, o grupo experimental recebia outra intervenção em conjunto com a música; e em 
dois $^{52,53}$, o grupo experimental tinha outra doença além de câncer. Dessa forma, permaneceram no estudo oito artigos para a análise qualitativa, conforme demonstrado na Figura 1.

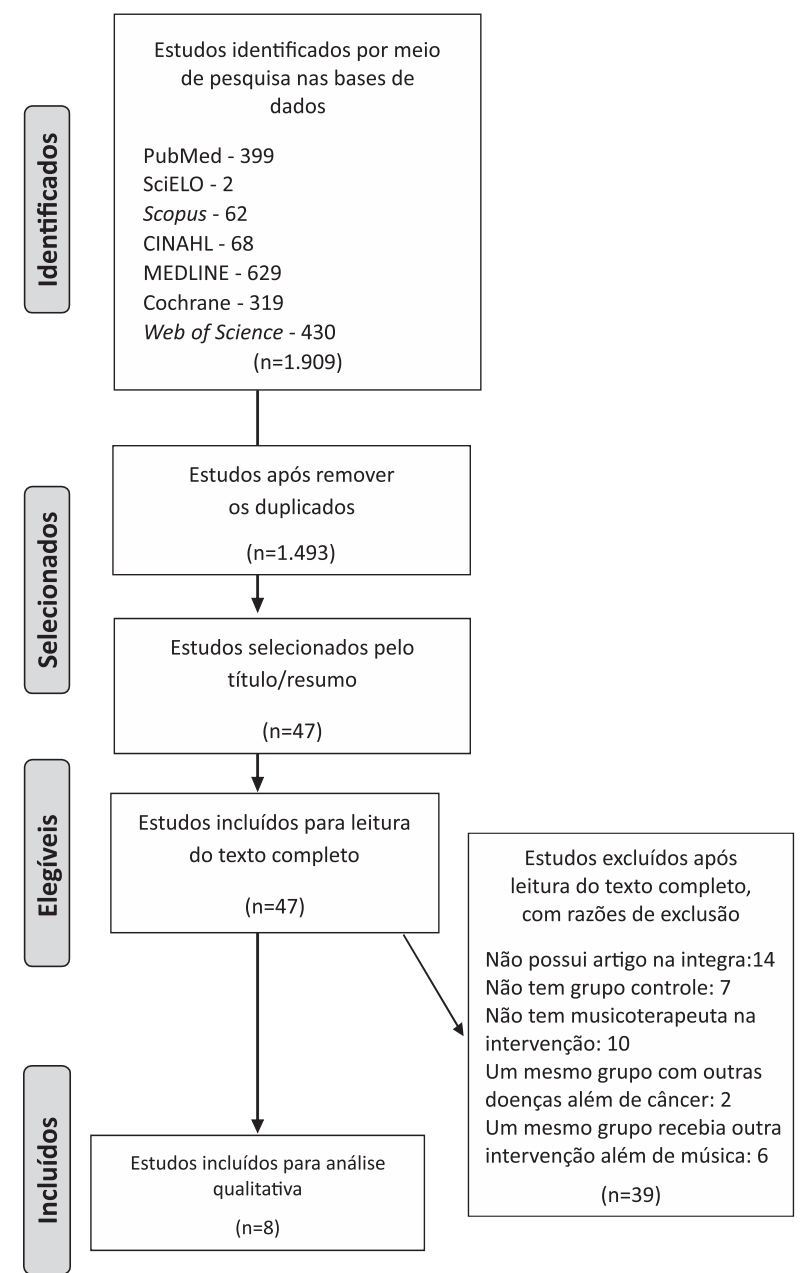

Figura 1. Fluxograma do estudo-Modelo PRISMA

Foram incluídos oito estudos que examinaram os efeitos da musicoterapia sobre a ansiedade em participantes com câncer. Os estudos apresentaram entre 33 a 68 participantes expostos à audição musical, que foram publicados no período de 2003 a $2017^{10-14,54-56} \mathrm{e}$ com total de 788 participantes (incluindo GE e GC). Três estudos $^{14,54,55}$ avaliaram a ansiedade com o instrumento State Trait Anxiety Inventory, dois estudos ${ }^{11,13}$ avaliaram com Hospital Anxiety and Depression Scale (HADS), um estudo ${ }^{10} \mathrm{com}$ Profile of Mood States (POMS), um estudo ${ }^{12}$ com Global Anxiety-Visual Analogic e um estudo ${ }^{56}$ com Visual Analogic Scale (VAS) (Tabela 1).

Dois tipos de mecanismos de oferta de música foram identificados: audiçáo de música com fones de ouvido $^{11,12,54,55}$ e audição com música ao vivo ${ }^{10,13,14,56}$.
A maioria dos estudos analisados selecionou a música com base nas preferências do paciente ${ }^{10-12,14,56}$. Quatro estudos ${ }^{10-12,55}$ descreveram a música utilizada como "relaxantes", "calmantes", "ativadoras"; dois ${ }^{54,56}$ utilizaram músicas instrumentais; e dois ${ }^{12,13}$ não descreveram o tipo de música utilizada.

A duração da intervenção musical foi descrita em seis estudos ${ }^{10,11,13,54-56}$, variando de $20^{13}$ a 90 minutos $^{11}$. Em dois estudos, a duração da intervenção musical foi descrita como compatível com o tempo do procedimento que estava sendo realizado ${ }^{12,14}$. A frequência de sessóes de musicoterapia foi de uma sessão $0^{54}$, duas sessóes por dia ${ }^{55}$, duas sessôes por semana ${ }^{13,56}$, variando o tempo entre os pacientes ${ }^{10}$ e sem detalhamento do número de sessóes realizadas na intervenção $\mathrm{O}^{11,12,14}$.

A escuta de música foi avaliada durante a radioterapia $^{11,14}$, quimioterapia ${ }^{13,54}, \operatorname{cirurgia}^{12,55} \mathrm{e}$ transplante ${ }^{10,56}$.

A amostra dos estudos foi composta principalmente por participantes com câncer de mama, sendo envolvidos também participantes com câncer hematológico, de pulmão, cabeça e pescoço.

\section{RESULTADOS}

A forma como a escolha da música foi realizada, pelo paciente ou pelo terapeuta, pareceu náo influenciar nos resultados, já que houve efeitos significativos da música na ansiedade nas duas formas de escolha. Entre os estudos que encontraram resultados significativos, em dois, a música foi selecionada pela preferência do paciente $^{14,57}$, em dois pela seleção do musicoterapeuta ${ }^{54,55}$, e em um ${ }^{12}$ pela preferência do paciente e por escolha do musicoterapeuta. Logo, a preferência musical não mostrou impactar nos benefícios da musicoterapia no tratamento para a ansiedade.

O tipo de execução da música, ao vivo ou pré-gravada, pareceu não influenciar nos efeitos da musicoterapia na ansiedade, já que, entre os estudos que encontraram efeitos na ansiedade, dois ${ }^{54,55}$ foram com música pré-gravada, dois com música ao vivo ${ }^{14,56} \mathrm{e}$ um com música gravada e ao vivo. Em relação ao tempo de música ofertado, houve variaçáo de $30^{56,57}$ a 60 minutos ${ }^{55}$ de intervenção musical nos estudos que observaram efeitos significativos.

Houve efetividade da musicoterapia na redução da ansiedade em diversos momentos de avaliaçáo: radioterapia ${ }^{14}$, quimioterapia ${ }^{54}$, cirurgia $^{12,55} \mathrm{e} \mathrm{transplante}^{56}$.

O risco de viés dos artigos incluídos foi detalhado nas Figuras 2 e 3.

Nos estudos avaliados, a principal razão para receber um alto risco de avaliaçáo de viés foi a falta de cegamento. O cegamento dos participantes é algo complexo em 
Tabela 1. Características dos estudos incluídos

\begin{tabular}{|c|c|c|c|c|c|c|c|c|}
\hline Autor/ano & Título & Amostra n & $\begin{array}{l}\text { Diagnóstico } \\
\text { da amostra }\end{array}$ & $\begin{array}{l}\text { Momento da } \\
\text { intervenção }\end{array}$ & $\begin{array}{l}\text { Execuçáo da } \\
\text { música }\end{array}$ & $\begin{array}{l}\text { Instrumento } \\
\text { de avaliação }\end{array}$ & Intervenção & Resultados \\
\hline $\begin{array}{l}\text { Cassileth et } \\
\text { al., } 2003\end{array}$ & $\begin{array}{l}\text { Music therapy for } \\
\text { mood disturbance } \\
\text { during } \\
\text { hospitalization } \\
\text { for autologous } \\
\text { stem cell } \\
\text { transplantation: } \\
\text { a randomized } \\
\text { controlled trial }\end{array}$ & $\begin{array}{l}\text { Total }=62 \\
\text { GE } n=34 \\
G C n=28\end{array}$ & $\begin{array}{c}\text { Câncer } \\
\text { hematológico } \\
\text { (linfoma não } \\
\text { Hodgkin, } \\
\text { linfoma de } \\
\text { Hodgkin, } \\
\text { mieloma } \\
\text { múltiplo) }\end{array}$ & $\begin{array}{l}\text { Durante } \\
\text { internação por } \\
\text { transplante } \\
\text { autólogo de } \\
\text { células-tronco }\end{array}$ & Música ao vivo & $\begin{array}{l}\text { Profile of } \\
\text { Mood States }\end{array}$ & $\begin{array}{l}\text { GE: sessão de } \\
\text { musicoterapia } \\
\text { Tipo de música: } \\
\text { preferências } \\
\text { musicais e } \\
\text { problemas clínicos } \\
\text { Duração: } \\
20 \text { a } 30 \text { min } \\
\text { GC: Cuidado padrão }\end{array}$ & $\begin{array}{c}\text { Sem mudanças } \\
\text { significativas na } \\
\text { ansiedade. GE } \\
\text { teve pontuação } \\
28 \% \text { menor na } \\
\text { escala combinada } \\
\text { de ansiedade/ } \\
\text { depressão (p } \\
0,065 \text { ), o humor } \\
\text { total teve melhora } \\
\text { significativa (p 0,01) } \\
\text { no GE em relação } \\
\text { ao GC }\end{array}$ \\
\hline $\begin{array}{l}\text { Clark et al., } \\
2006\end{array}$ & $\begin{array}{l}\text { Use of preferred } \\
\text { music to reduce } \\
\text { emotional distress } \\
\text { and symptom } \\
\text { activity during } \\
\text { radiation therapy }\end{array}$ & $\begin{array}{l}\text { Total }=63 \\
\text { GE } n=35 \\
G C n=28\end{array}$ & $\begin{array}{c}\text { Câncer de } \\
\text { vários tipos e } \\
\text { locais variados }\end{array}$ & $\begin{array}{c}\text { Durante } \\
\text { Radioterapia }\end{array}$ & $\begin{array}{l}\text { Música gravada } \\
\text { tocando } \\
\text { audição por } \\
\text { fone de ouvido } \\
\text { conectado ao } \\
\text { toca-fitas }\end{array}$ & $\begin{array}{l}\text { Hospital } \\
\text { Anxiety and } \\
\text { Depression } \\
\text { Scale }\end{array}$ & $\begin{array}{l}\text { GE: sessão de } \\
\text { musicoterapia } \\
\text { Tipo de música: } \\
\text { estilos musicais } \\
\text { preferidos } \\
\text { pelos pacientes } \\
\text { identificados pelo } \\
\text { musicoterapeuta } \\
\text { para uso como } \\
\text { relaxamento ou } \\
\text { distração } \\
\text { Duração: } 90 \text { min } \\
\text { GC: Cuidados usuais }\end{array}$ & $\begin{array}{c}\text { Ansiedade } \\
\text { e angústia } \\
\text { diminuiram no GC, } \\
\text { mas sem resultado } \\
\text { significativo. A } \\
\text { única correlação } \\
\text { significativa } \\
\text { foi entre uso } \\
\text { de música e } \\
\text { sofrimento } \\
\text { (r=0,48, } \\
\mathrm{p}=0,02)_{1} \\
\text { indicando maior } \\
\text { uso de música, } \\
\text { maior redução } \\
\text { no sofrimento } \\
\text { relacionado ao } \\
\text { tratamento }\end{array}$ \\
\hline $\begin{array}{l}\text { Lin et al., } \\
2011\end{array}$ & $\begin{array}{l}\text { A randomised } \\
\text { controlled trial } \\
\text { of the effect of } \\
\text { music therapy } \\
\text { and verbal } \\
\text { relaxation on } \\
\text { chemotherapy- } \\
\text { induced anxiety }\end{array}$ & $\begin{array}{c}\text { Total }=98 \\
\text { GE } n=34 \\
G C n=34 \\
\text { Relaxamento } \\
n=30\end{array}$ & $\begin{array}{l}\text { Câncer de } \\
\text { mama, } \\
\text { pulmão e } \\
\text { outros }\end{array}$ & $\begin{array}{c}\text { Durante } \\
\text { quimioterapia }\end{array}$ & $\begin{array}{l}\text { Música gravada } \\
\text { - audição por } \\
\text { fone de ouvido } \\
\text { conectado ao } \\
\text { MP3 player }\end{array}$ & $\begin{array}{c}\text { State Trait } \\
\text { Anxiety } \\
\text { Inventory }\end{array}$ & $\begin{array}{l}\text { GE: sessão de } \\
\text { musicoterapia } \\
\text { Tipo de música: } \\
\text { 1. preparação - sons } \\
\text { da natureza; } 2 \text {. } \\
\text { relaxamento - } \\
\text { meditação e sons } \\
\text { da natureza; } 3 \text {. } \\
\text { audição de música } \\
\text { instrumental e da } \\
\text { natureza } \\
\text { Duração: } 60 \text { min } \\
\text { GC: recebeu cuidados } \\
\text { de enfermagem de } \\
\text { rotina }\end{array}$ & $\begin{array}{l}\text { A intervenção } \\
\text { musical durante } \\
\text { um protocolo de } \\
\text { quimioterapia } \\
\text { de } 30 \text { minutos } \\
\text { possui efeitos } \\
\text { significativos } \\
\text { na redução da } \\
\text { ansiedade em } \\
\text { pacientes com } \\
\text { ansiedade elevada } \\
\text { pré-quimioterapia } \\
(p=0,028)\end{array}$ \\
\hline Li et al., 2011 & $\begin{array}{l}\text { Effects of } \\
\text { music therapy } \\
\text { on anxiety of } \\
\text { patients with } \\
\text { breast cancer } \\
\text { after radical } \\
\text { mastectomy: } \\
\text { a randomized } \\
\text { clinical trial }\end{array}$ & $\begin{array}{c}\text { Total }=120 \\
G E n=60 \\
G C n=60\end{array}$ & $\begin{array}{c}\text { Câncer de } \\
\text { mama }\end{array}$ & $\begin{array}{l}\text { Durante pós- } \\
\text { operatório de } \\
\text { mastectomia } \\
\text { radical }\end{array}$ & $\begin{array}{l}\text { Música gravada } \\
\text { - audição por } \\
\text { fone de ouvido } \\
\text { conectado ao } \\
\text { MP3 player }\end{array}$ & $\begin{array}{c}\text { State Trait } \\
\text { Anxiety } \\
\text { Inventory }\end{array}$ & $\begin{array}{l}\text { GE: sessão de } \\
\text { musicoterapia duas } \\
\text { vezes ao dia, uma } \\
\text { vez no início da } \\
\text { manhã e outra à } \\
\text { noite, e cuidados de } \\
\text { enfermagem de rotina } \\
\text { Tipo de música: } \\
\text { pré-selecionadas } \\
202 \text { músicas por } \\
\text { profissionais depois } \\
\text { de pacientes escolhem } \\
\text { suas preferidas } \\
\text { Duração: } 30 \text { min } \\
\text { GC: apenas cuidados } \\
\text { de enfermagem de } \\
\text { rotina }\end{array}$ & $\begin{array}{c}\text { Os escores de } \\
\text { ansiedade } \\
\text { do GE foram } \\
\text { significativamente } \\
\text { menores do que } \\
\text { do GC em cada um } \\
\text { dos três pós-testes } \\
\text { ( } \mathrm{p}<0,0001)\end{array}$ \\
\hline
\end{tabular}


Tabela 1. continuação

\begin{tabular}{|c|c|c|c|c|c|c|c|c|}
\hline Autor/ano & Título & Amostra n & $\begin{array}{l}\text { Diagnóstico } \\
\text { da amostra }\end{array}$ & $\begin{array}{l}\text { Momento da } \\
\text { intervenção }\end{array}$ & $\begin{array}{l}\text { Execuçáo da } \\
\text { música }\end{array}$ & $\begin{array}{l}\text { Instrumento } \\
\text { de avaliação }\end{array}$ & Intervenção & Resultados \\
\hline $\begin{array}{l}\text { Palmer et al., } \\
2015\end{array}$ & $\begin{array}{l}\text { Effects of } \\
\text { music therapy } \\
\text { on anesthesia } \\
\text { requirements and } \\
\text { anxiety in women } \\
\text { undergoing } \\
\text { ambulatory } \\
\text { breast surgery for } \\
\text { cancer diagnosis } \\
\text { and treatment: } \\
\text { a randomized } \\
\text { controlled trial }\end{array}$ & $\begin{array}{c}\text { Total }=201 \\
\text { Música ao vivo } \\
n=68 \\
\text { Música } \\
\text { gravada } \\
n=68 \\
G C n=65\end{array}$ & $\begin{array}{l}\text { Câncer de } \\
\text { mama }\end{array}$ & $\begin{array}{c}\text { Durante pré- } \\
\text {-operatório e } \\
\text { intraoperatório } \\
\text { de cirurgia de } \\
\text { mama }\end{array}$ & $\begin{array}{l}\text { Música ao vivo e } \\
\text { música gravada } \\
\text { audição por } \\
\text { fone de ouvido } \\
\text { conectado ao } \\
\text { MP3 player }\end{array}$ & $\begin{array}{l}\text { Global Anxiety } \\
\text { - Visual Analog }\end{array}$ & $\begin{array}{l}\text { GM ao vivo: pré- } \\
\text {-operatório - música } \\
\text { ao vivo selecionada } \\
\text { pelo paciente } \\
\text { GM gravada: } \\
\text { intraoperatório - } \\
\text { música gravada } \\
\text { selecionada pelo } \\
\text { terapeuta } \\
\text { Duração: } \\
\text { tempo do } \\
\text { procedimento } \\
\text { GC: pré-operatório } \\
\text { - cuidados usuais; } \\
\text { intraoperatório } \\
\text { - protetores } \\
\text { bloqueadores de } \\
\text { ruídos }\end{array}$ & $\begin{array}{l}\text { Houve redução } \\
\text { significativa da } \\
\text { ansiedade nos } \\
\text { grupos de música } \\
\text { ao vivo e música } \\
\text { gravada se } \\
\text { comparada ao GC } \\
\text { de cuidados usuais } \\
\text { ( }<<0,001 \text { ) } \\
\text { Não houve } \\
\text { diferença } \\
\text { significativa na } \\
\text { comparação do GM } \\
\text { ao vivo e música } \\
\text { gravada }\end{array}$ \\
\hline $\begin{array}{l}\text { Tuinmann et } \\
\text { al., } 2016\end{array}$ & $\begin{array}{l}\text { The effects of } \\
\text { music therapy } \\
\text { in patients } \\
\text { with high-dose } \\
\text { chemotherapy } \\
\text { and stem cell } \\
\text { support: a } \\
\text { randomized pilot } \\
\text { study }\end{array}$ & $\begin{array}{l}\text { Total }=66 \\
\text { GE } n=33 \\
G C n=33\end{array}$ & $\begin{array}{c}\text { Câncer } \\
\text { hematológico } \\
\text { (linfoma não } \\
\text { Hodgkin, } \\
\text { linfoma de } \\
\text { Hodgkin, } \\
\text { mieloma } \\
\text { múltiplo, } \\
\text { câncer de } \\
\text { testículo e } \\
\text { leucemia) }\end{array}$ & $\begin{array}{c}\text { Durante } \\
\text { quimioterapia }\end{array}$ & $\begin{array}{l}\text { Música ao vivo } \\
\text { Métodos ativos } \\
\text { (tocar música } \\
\text { e cantar) ou } \\
\text { receptivos (ouvir } \\
\text { música), de } \\
\text { acordo com as } \\
\text { necessidades } \\
\text { terapêuticas }\end{array}$ & $\begin{array}{c}\text { Hospital } \\
\text { Anxiety and } \\
\text { Depression } \\
\text { Scale }\end{array}$ & $\begin{array}{l}\text { GE: sessão de } \\
\text { musicoterapia duas } \\
\text { vezes por semana } \\
\text { Tipo de música: } \\
\text { intervenção não } \\
\text { foi planejada nem } \\
\text { padronizada } \\
\text { Duração: } \\
20 \text { min } \\
\text { GC: tratamento } \\
\text { padrão } \\
\text { (atendimento } \\
\text { profissional } \\
\text { por médicos e } \\
\text { enfermeiros) }\end{array}$ & $\begin{array}{l}\text { A redução da } \\
\text { ansiedade não } \\
\text { foi significativa } \\
\text { ( } p=0,722) \\
\text { Em ambos os } \\
\text { grupos, } 0 \text { escore } \\
\text { de ansiedade } \\
\text { diminuiu. Como } \\
\text { a pontuação } \\
\text { média de ambos } \\
\text { os grupos não } \\
\text { excedia o ponto } \\
\text { de corte, esses } \\
\text { achados não } \\
\text { são clinicamente } \\
\text { relevantes }\end{array}$ \\
\hline $\begin{array}{l}\text { Dóro et al., } \\
2016\end{array}$ & $\begin{array}{l}\text { Music therapy } \\
\text { improves the } \\
\text { mood of patients } \\
\text { undergoing } \\
\text { hematopoietic } \\
\text { stem cells } \\
\text { transplantation } \\
\text { (controlled } \\
\text { randomized } \\
\text { study) }\end{array}$ & $\begin{array}{c}\text { Total }=100 \\
\text { GE } n=50 \\
G C n=50\end{array}$ & $\begin{array}{c}\text { Câncer } \\
\text { hematológico }\end{array}$ & $\begin{array}{l}\text { Na enfermaria } \\
\text { com pacientes } \\
\text { submetidos a } \\
\text { transplante } \\
\text { alogênico de } \\
\text { células-tronco } \\
\text { hematopoiéticas }\end{array}$ & $\begin{array}{c}\text { Música ao } \\
\text { vivo Paciente } \\
\text { e terapeuta } \\
\text { executam } \\
\text { e cantam } \\
\text { músicas pré- } \\
\text {-determinadas } \\
\text { em instrumentos, } \\
\text { improvisam } \\
\text { músicas } \\
\text { espontaneamente } \\
\text { usando a voz, } \\
\text { instrumentos ou } \\
\text { ambos }\end{array}$ & $\begin{array}{l}\text { Visual Analog } \\
\text { Scale (VAS) }\end{array}$ & $\begin{array}{l}\text { GE: sessão de } \\
\text { musicoterapia duas } \\
\text { vezes por semana } \\
\text { Tipo de música: } \\
\text { escolhidas pelos } \\
\text { terapeutas, músicas } \\
\text { populares que } \\
\text { fazem parte da } \\
\text { identidade sócio- } \\
\text {-musical-cultural do } \\
\text { paciente, e músicas } \\
\text { de preferência do } \\
\text { paciente } \\
\text { Duração: } \\
30 \text { min } \\
\text { GC: tratamento } \\
\text { padrão }\end{array}$ & $\begin{array}{c}\text { Redução } \\
\text { estatisticamente } \\
\text { significativa da } \\
\text { ansiedade } \\
(p<0,001)\end{array}$ \\
\hline $\begin{array}{l}\text { Rossetti et al., } \\
2017\end{array}$ & $\begin{array}{l}\text { The impact of } \\
\text { music therapy } \\
\text { on anxiety in } \\
\text { cancer patients } \\
\text { undergoing } \\
\text { simulation for } \\
\text { radiation therapy }\end{array}$ & $\begin{array}{c}\text { Total }=78 \\
\text { GE } n=39 \\
G C n=39\end{array}$ & $\begin{array}{l}\text { Câncer de } \\
\text { mama e de } \\
\text { cabeça e } \\
\text { pescoço }\end{array}$ & Radioterapia & Música ao vivo & $\begin{array}{c}\text { State Trait } \\
\text { Anxiety } \\
\text { Inventory }\end{array}$ & $\begin{array}{l}\text { GE: sessão de } \\
\text { musicoterapia } \\
\text { Tipo de música } \\
\text { com base nas } \\
\text { preferências do } \\
\text { paciente, músicas } \\
\text { ativadoras e } \\
\text { relaxantes } \\
\text { Duração: } \\
\text { Tempo de } \\
\text { procedimento } \\
\text { GC: tratamento } \\
\text { padrão com } \\
\text { instruções verbais e } \\
\text { instruções por vídeo }\end{array}$ & $\begin{array}{c}\text { Redução } \\
\text { significativa na } \\
\text { ansiedade nos } \\
\text { indivíduos do GE } \\
(\mathrm{p}<0,0001)\end{array}$ \\
\hline
\end{tabular}

Legendas: GC: gupo controle; GE: grupo experimental; GM: grupo de música. 
estudos de musicoterapia, nesse sentido, é difícil que estes recebam um risco de viés baixo ou moderado, mesmo que tenham abordado adequadamente todos os outros fatores de risco (por exemplo, randomização, ocultação de alocação etc.).

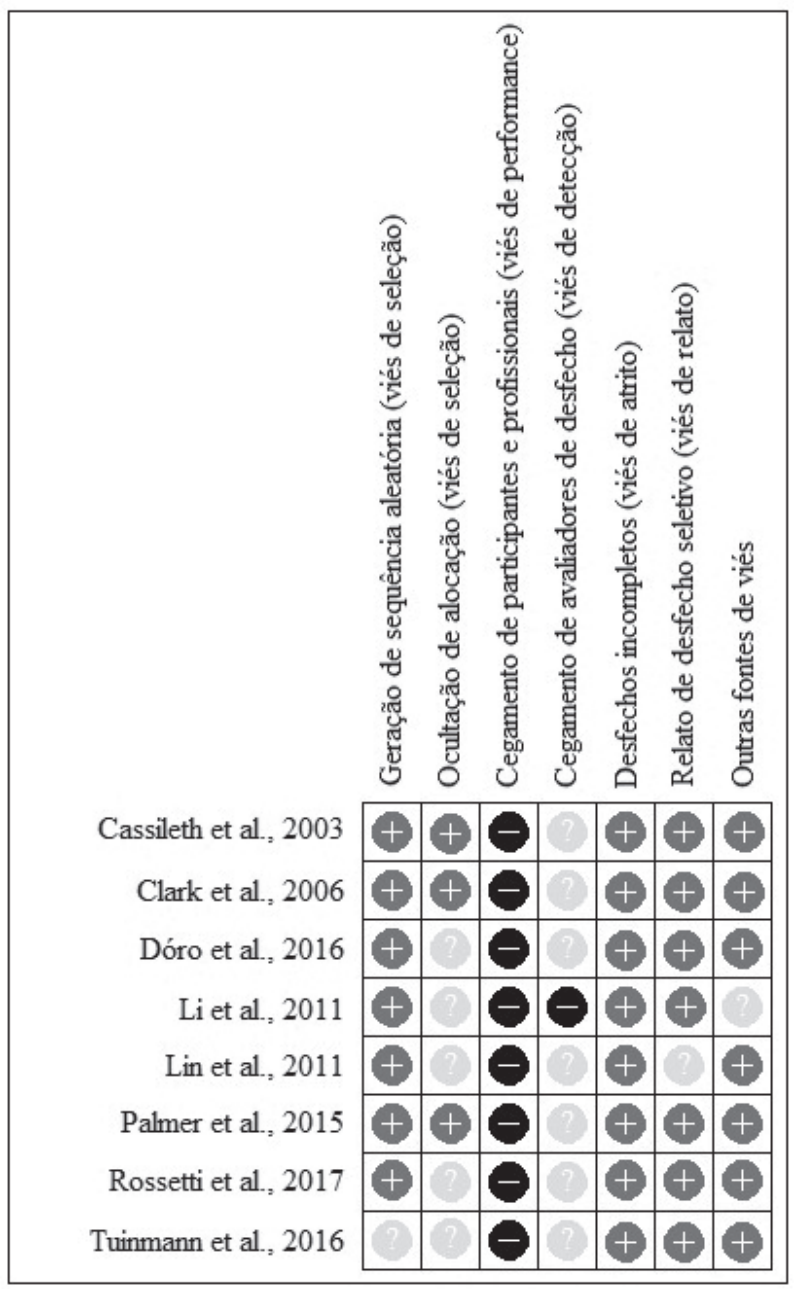

Figura 2. Risco de viés pela ferramenta Cochrane
Vale a pena notar que alguns estudos não forneceram informaçōes suficientes acerca do risco de viés. Não está claro, no entanto, se isso foi em razão das traduçōes incompletas ou da falta de detalhes nos relatórios originais do estudo.

Dos oito estudos selecionados, dois obtiveram nota 7 e seis nota 6 na Escala PEDro, demonstrando boa qualidade metodológica dos estudos avaliados (Tabela 2$)^{58}$.

\section{DISCUSSÃO}

A maioria dos ensaios clínicos avaliados sugere que a intervenção musical reduz a ansiedade em pessoas com câncer, em diversos momentos do tratamento, o que vai de acordo com achados da literatura que trazem o efeito da música na redução da ansiedade na radioterapia ${ }^{27,30,34}$, quimioterapia ${ }^{47,49,59,60}$, além de efeitos na ansiedade nas fases pré e pós-operatórias ${ }^{61}$. Uma das possíveis explicaçóes para esse fato é que a audição musical pode reduzir a secreção de catecolaminas na regulação das funçóes autonômicas e melhorar as respostas fisiológicas, como frequência respiratória, frequência cardíaca, pressão arterial, temperatura corporal e tensão muscular, o que causa efeitos no relaxamento e reduçáo da ansiedade ${ }^{61}$.

Os estudos que não apresentaram efeito significativo da musicoterapia na ansiedade justificam os resultados em virtude da náo apresentaçáo de um nível alto o suficiente de ansiedade no início do estudo. Acerca disso, é importante relatar a diferenciação entre estar ansioso e ser ansioso, que não foi discutida nos estudos. A ansiedade é uma condição clínica complexa, que pode ser dividida em estado de ansiedade e traço de ansiedade. $\mathrm{O}$ estado de ansiedade é definido como uma atividade emocional transitória, de acordo com as condiçóes momentâneas vivenciadas pelo indivíduo, surgindo sentimentos de tensão e apreensão conscientemente percebidos, que

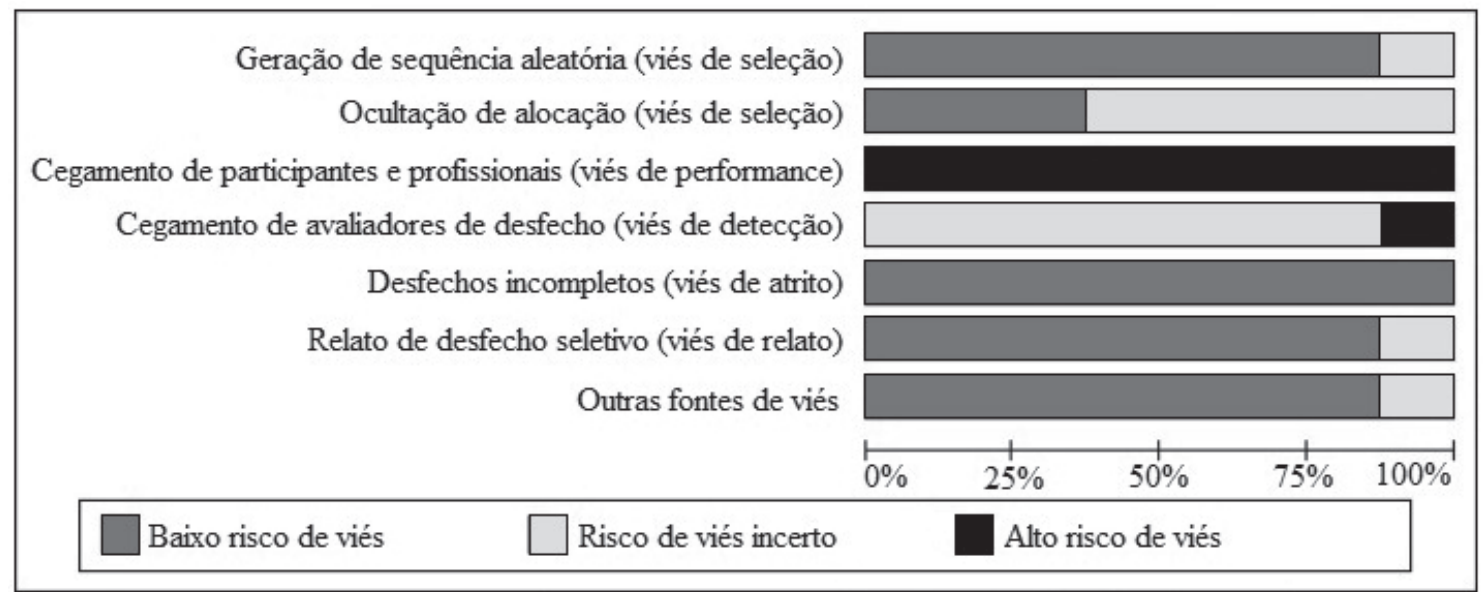

Figura 3. Resumo do risco de viés pela ferramenta Cochrane 
Tabela 2. Avaliação da qualidade metodológica dos estudos incluídos na revisão analisada pela escala PEDro

\begin{tabular}{l|l|l|l|l|l|l|l|l|l|c|c|c|}
\hline \multicolumn{1}{c|}{ Autor/ano } & $\mathbf{1}$ & $\mathbf{2}$ & $\mathbf{3}$ & $\mathbf{4}$ & $\mathbf{5}$ & $\mathbf{6}$ & $\mathbf{7}$ & $\mathbf{8}$ & $\mathbf{9}$ & $\mathbf{1 0}$ & $\mathbf{1 1}$ & Total \\
\hline Cassileth et al., 2003 & 1 & 1 & 1 & 1 & 0 & 0 & 0 & 1 & 1 & 1 & 1 \\
Clark et al., 2006 & 1 & 1 & 1 & 0 & 0 & 0 & 0 & 1 & 1 & 1 & 1 & 6 \\
Dóro et al., 2016 & 1 & 1 & 0 & 1 & 0 & 0 & 0 & 1 & 1 & 1 & 1 & 6 \\
Li et al., 2011 & 1 & 1 & 0 & 1 & 0 & 0 & 0 & 1 & 1 & 1 & 1 & 6 \\
Lin et al., 2011 & 0 & 1 & 0 & 1 & 0 & 0 & 0 & 1 & 1 & 1 & 1 & 6 \\
Palmer et al., 2015 & 1 & 1 & 1 & 1 & 0 & 0 & 0 & 1 & 1 & 1 & 1 & 7 \\
Rossetti et al., 2017 & 1 & 1 & 0 & 1 & 0 & 0 & 0 & 1 & 1 & 1 & 1 & 6 \\
Tuinmann et al., 2016 & 1 & 1 & 0 & 1 & 0 & 0 & 0 & 1 & 1 & 1 & 1 & 6 \\
\hline
\end{tabular}

Legendas: 1 Eligibility criteria, 2 Random allocation, 3 Concealed allocation, 4 Baseline Comparability, 5 Blind subjects, 6 Blind therapists, 7 Blind assessor, $8<15 \%$ of desistence, 9 Intention to treat analysis, 10 Between groups comparison, 11 Point estimates and variability.

Nota: Criterion 1 is not considered for the final score because it is an item that assesses the external validity ${ }^{58}$.

podem variar de intensidade. Já o traço de ansiedade é como a pessoa costuma reagir frente às situaçóes de estresse do cotidiano e que são percebidas como ameaçadoras, sendo que o traço de ansiedade é estável, permanente, variando de acordo com cada indivíduo ${ }^{62}$. Sendo assim, a amostra dos estudos poderia ser melhor classificada considerando esses aspectos. Estudos relatam que pacientes com níveis mais altos de ansiedade ou angústia respondem mais favoravelmente à musicoterapia do que pacientes com níveis mais baixos de sofrimento, sendo assim a falta de homogeneidade dos grupos de acordo com o nível de ansiedade pode ter influenciado na falta de significância de alguns resultados ${ }^{11}$.

Em quatro ${ }^{12,14,54,55}$ dos cinco estudos que apresentaram significância do efeito da musicoterapia, o sexo feminino foi maioria na amostra. Sabe-se que em torno de $25 \%$ a $35 \%$ das mulheres com câncer irão desenvolver ansiedade e/ou depressão em algum estágio do tratamento ${ }^{63}$. Nesse sentido, é necessário compreender os aspectos que podem favorecer tal diferença entre os sexos, uma vez que a literatura traz a relação entre o câncer de mama com maior presença de distúrbios emocionais ${ }^{33,64-66}$. Os resultados de um dos estudos incluídos nessa revisão ${ }^{55}$, com amostra composta por 120 pacientes exclusivamente do sexo feminino, trouxeram que o pré-teste do estado de ansiedade demonstrava que a maioria dos pacientes apresentava ansiedade moderada e grave, indicando que é uma distúrbio psicológico frequente em mulheres com câncer. A maior parte da amostra dos artigos incluídos foi composta por participantes com câncer de mama, um dos mais incidentes no sexo feminino. Nesse tipo de câncer, o principal tratamento é a cirurgia, na qual a mama pode ser retirada total ou parcialmente. Ter a mama atingida pela doença e mutilada pelo tratamento pode interferir na identidade feminina, levando a intenso sofrimento psicológico, o qual ultrapassa o da doença em si, pois provoca alteração da imagem corporal, gera fragilidade emocional, baixa autoestima, medo da rejeição e comprometimento no relacionamento interpessoal e social $^{67}$. Relatos demonstram alta prevalência de ansiedade, por pelo menos dois anos após o diagnóstico, em uma a cada cinco mulheres com câncer de mama ${ }^{3}$. Novas revisóes que analisem estudos com amostras separadas entre mulheres e homens podem trazer melhor confirmação da associaçáo da efetividade da musicoterapia em ambos os sexos.

A maior parte dos estudos analisados selecionou a música com base nas preferências do paciente ${ }^{10-12,14,56}$. Um dos estudos ${ }^{12}$ incluídos nessa revisão relatou que melodias familiares ou de preferência do paciente seriam mais benéficas, justificando que isso acontece uma vez que os sintomas da ansiedade se exacerbam geralmente quando o que é esperado diverge do que é real. Sendo assim, proporcionar o que é esperado, conhecido (a música preferida), pode estimular a resposta de relaxamento por meio da ativação do ramo parassimpático do sistema nervoso autônomo. Melodias familiares, padróes rítmicos e letras de músicas podem fornecer um contraste bem-vindo ao sofrimento, proporcionando o previsível em um ambiente imprevisível, aumentando os fatores de conforto, de um tratamento ainda desconhecido, restaurando, assim, o equilíbrio do sistema nervoso autônomo. No entanto, os resultados desta revisão mostraram que a musicoterapia foi efetiva no manejo da ansiedade, independentemente de a música ser escolhida ou não pelo paciente.

A música é uma forma complexa e subjetiva de tratamento, o que justifica a alta heterogeneidade de intervençóes relatadas nos estudos incluídos. Nesse contexto, algumas variáveis náo padronizadas podem ter influenciado o efeito estatístico dos estudos, como: não diferença entre o efeito da música e o efeito do profissional musicoterapeuta, bem como diferentes ambientes clínicos, tipos de intervenção; instrumentos utilizados, momentos da avaliação, duração da intervenção, duração dos benefícios e tipo de música selecionada, o que dificulta a realização de comparaçóes. Além disso, a descrição da música em diferentes termos carece interpretação que universalize as intervençóes. Isso reforça a necessidade 
de uma abordagem mais objetiva e específica, com uma descrição apropriada da intervenção musical, como forma de facilitar a reprodutividade dos estudos na prática clínica.

\section{CONCLUSÃO}

A musicoterapia é efetiva na redução da ansiedade de pessoas com câncer. No entanto, mais estudos com novas tecnologias e mais detalhes sobre a intervenção são necessários para a confirmação dos resultados.

\section{CONTRIBUIÇÕES}

Todos os autores contribuíram substancialmente na concepçáo e no planejamento do estudo, na obtençáo, análise e interpretação dos dados, na redação e/ ou revisão crítica e aprovação final da versão publicada.

\section{DECLARAÇÃO DE CONFLITOS DE INTERESSES}

Nada a declarar.

\section{FONTES DE FINANCIAMENTO}

Não há.

\section{REFERÊNCIAS}

1. Mitchell AJ, Chan M, Bhatti H, et al. Prevalence of depression, anxiety, and adjustment disorder in oncological, haematological, and palliative-care settings: a meta-analysis of 94 interview-based studies. Lancet Oncol. 2011;12(2):160-741. doi: https://doi. org/10.1016/S1470-2045(11)70002-X

2. Zeynalova N, Schimpf S, Setter C, et al. The association between an anxiety disorder and cancer in medical history. J Affect Disord. 2019;246:640-2. doi: https:// doi.org/10.1016/j.jad.2018.12.019

3. Carreira H, Williams R, Müller M, et al. Associations between breast cancer survivorship and adverse mental health outcomes: a systematic review. J Natl Cancer Inst. 2018;110(12):1311-27. doi: https://doi.org/10.1093/ jnci/djy177

4. Grandizoli MV, Ibiapina ISM, Santos Junio R, et al. Indicadores de esperança, ansiedade e depressão de pacientes em tratamento oncológico. Arq Ciênc Saúde. 2017;24(3):65-70. doi: https://doi.org/10.17696/23183691.24.3.2017.718

5. Beaudreau SA, MacKay-Brandt A, Reynolds J. Application of a cognitive neuroscience perspective of cognitive control to late-life anxiety. J Anxiety Disord. 2013;27(6):559-66. doi: https://doi.org/10.1016/j. janxdis.2013.03.006
6. Martin-Saavedra JS, Vergara-Mendez LD, TaleroGutiérrez C. Music is an effective intervention for the management of pain: an umbrella review. Complement Ther Clin Pract. 2018;32:103-14. doi: https://doi. org/10.1016/j.ctcp.2018.06.003

7. Gallagher LM, Lagman R, Rybicki L. Outcomes of music therapy interventions on symptom management in palliative medicine patients. Am J Hosp Palliat Care. 2018;35(2):250-7. doi: https://doi. org/10.1177/1049909117696723

8. Stanczyk MM. Music therapy in supportive cancer care. Rep Pract Oncol Radiother. 2011;16(5):170-2. doi: http://dx.doi.org/10.1016/j.rpor.2011.04.005

9. Nilsson U. Soothing music can increase oxytocin levels during bed rest after open-heart surgery: a randomised control trial. J Clin Nurs. 2009;18(15):2153-61. doi: https://doi.org/10.1111/j.1365-2702.2008.02718.x

10. Cassileth BR, Vickers AJ, Magill LA. Music therapy for mood disturbance during hospitalization for autologous stem cell transplantation: a randomized controlled trial. Cancer. 2003;98(12):2723-9. doi: https://doi. org/10.1002/cncr.11842

11. Clark M, Isaacks-Downton G, Wells N, et al. Use of preferred music to reduce emotional distress and symptom activity during radiation therapy. J Music Ther. 2006;43(3):247-65. doi: https://doi.org/10.1093/ jmt/43.3.247

12. Palmer JB, Lane D, Mayo D, et al. Effects of music therapy on anesthesia requirements and anxiety in women undergoing ambulatory breast surgery for cancer diagnosis and treatment: a randomized controlled trial. J Clin Oncol. 2015 Oct 1;33(28):3162-8. doi: https:// doi.org/10.1200/JCO.2014.59.6049

13. Tuinmann G, Preissler P, Böhmer H, et al. The effects of music therapy in patients with high-dose chemotherapy and stem cell support: a randomized pilot study. Psychooncology. 2016 May;26(3):377-84. doi: https:// doi.org/10.1002/pon.4142

14. Rossetti A, Chadha M, Torres BN, et al. The impact of music therapy on anxiety in cancer patients undergoing simulation for radiation therapy. Int J Radiat Oncol Biol Phys. 2017;99(1):103-10. doi: http://dx.doi. org/10.1016/j.ijrobp.2017.05.003

15. Cai GR, Li PW, Jiao LP. Clinical observation of music therapy combined with anti-tumor drugs in treating 116 cases of tumor patients. Zhongguo Zhong xi yi jie he za zhi [Internet]. 2001 Dec [cited 2019 Oct 15];21(12):891-4. Available from: https://www.ncbi. nlm.nih.gov/pubmed/12575587 China. Cited in: PubMed; PMID 12575587.

16. Determann MM, Kalthoff H, Kollenbaum VE, et al. First results of a prospective randomized study on the impact of psychooncological intervention in cancer patients on quality of life and endocrino-immunological parameters. 
Eur-j-cancer. 2001;37(Suppl 6):S162 Abs.589. doi: https://doi.org/10.1016/S0959-8049(01)81081-9

17. Toole M, Bendinger GM, Ensor JE, et al. A randomized study of personalized music therapy for patients with early stage breast cancer receiving chemotherapy [abstract OT3-08-01]. Cancer Res. 2017;77(4 Suppl). From a paper presented at the 39th Annual CTRC AACR San Antonio Breast Cancer Symposium, San Antonio, TX, December 6-10, 2016. doi: https://doi. org/10.1158/1538-7445.SABCS16-OT3-08-01

18. Mantovan F, Rauter E, Müller I. Massagen und musiktherapie zur reduktion der angst von onkologischen patienten bei der palliativversorgung [Massage and music therapy for relief of anxiety of cancer patients in palliative care]. Pflege Z [Internet]. 2009 [cited 2019 Oct 17];62(3):164-9. Available from: http://pesquisa. bvsalud.org/portal/resource/pt/mdl-19348407 German

19. Beth Israel Medical Center. Impact of music therapy on anxiety in patients with cancer undergoing simulation for radiation therapy. 2011 July [last updated 2014 May 29; cited 2019 Oct 20. In: ClinicalTrials.gov [Internet]. Bethesda (MD): U.S. National Library of Medicine. 2000 - . Available from: https://clinicaltrials. gov/ct2/show/study/NCT02150395. ClinicalTrials.gov Identifier: NCT 02150395.

20. Wake Forest University Health Sciences. Reducing lung cancer-related anxiety (relax). 2015 July 30 [first Posted 2014 Feb 14; cited 2019 Oct 20]. In: ClinicalTrials.gov [Internet]. Bethesda (MD): U.S. National Library of Medicine. 2000 -. Available from: https://clinicaltrials. gov/ct2/show/record/NCT02063828 ClinicalTrials.gov Identifier: NCT02063828.

21. Massimiliani V, Pellegrino R, Donnarumma L, et al. Music intervention during chemotherapy infusion reduces anxiety in oncological patients. Ann Oncol. 2017;28(Suppl 6):vi82-vi88. From a paper presented at the 19th National Congress of Medical Oncology, Rome, ITA, 2017 Oct 27-29. doi: https://doi.org/10.1093/ annonc/mdx 434.002

22. Hanser S. Evidence-based music therapy protocols in integrative medicine and health. BMC Complement Altern Med. 2017, 17(Suppl):P317. From a paper presented at the World Congress Integrative Medicine and Health, Berlin, DEU, 2017 May 3. doi: https://doi. org/10.1186/s12906-017-1784-2

23. Case Comprehensive Cancer Center. Music therapy and hematopoietic stem cell transplant. 2018 Feb 26 [first Posted 2017 Dec 19; Last Update Posted 2019 Aug 1; cited 2019 Oct 21]. In: ClinicalTrials.gov [Internet]. Bethesda (MD): U.S. National Library of Medicine. 2000 - . Available from: https://clinicaltrials. gov/show/nct03378089 ClinicalTrials.gov Identifier: NCT03378089.

24. University of Florida. Music of choice to decrease anxiety during radiation treatment. 2018 Jan 22 [First Posted
2018 May 147; cited 2019 Oct 21]. In: ClinicalTrials. gov [Internet]. Bethesda (MD): U.S. National Library of Medicine. 2000 - . Available from: https://clinicaltrials. gov/ct2/show/record/NCT03527225 ClinicalTrials.gov Identifier: NCT03527225.

25. Rossetti A, Chadha M, Lucido D, et al. The impact of music therapy on anxiety and distress in patients undergoing simulation for radiation therapy (RT). Int J Radiat Oncol Biol Phys. 2014;90(1 Suppl 1):S708-S709. From a paper presented at the 56th Annual Meeting of the American Society for Radiation Oncology, ASTRO 2014 San Francisco, CA United States. doi: https://doi. org/10.1016/j.ijrobp.2014.05.2074

26. Hanser SB, Bauer-Wu S, Kubicek L, et al. a Effects of a music therapy intervention on quality of life and distress in women with metastatic breast cancer. J Soc Integr Oncol. 2006;4(3):116-24. doi: https://doi. org/10.2310/7200.2006.014

27. Zhao PT, Liang J, Shao QJ, et al. Interventional effects of musical therapy to physiological and psychological conditions in process of radiotherapy for patients with cancer. Chinese J Cancer Prev Treat [Internet]. 2008 [cited 2019 Oct 22];15(14):1097-99. Available from: https://www.cochranelibrary.com/central/doi/10.1002/ central/CN-00708265/full

28. Tan BL, Sin ACF, Ho SM, et al. Effect of music in reducing anxiety levels among patients who receive their first dose of chemotherapy treatment. Singapore Gen Hosp Proc [Internet]. 2008 [cited 2019 Oct 22];17(1):46-56. Available from: https://www.cochranelibrary.com/ central/doi/10.1002/central/CN-00707443/full

29. Firmeza MA, Rodrigues AB, Melo GAA, et al. Control of anxiety through music in a head and neckoutpatient clinic: a randomized clinical trial. Rev Esc Enferm USP. 2017;51:e03201. doi: http://dx.doi.org/10.1590/s1980220x2016030503201

30. Hanedan Uslu G. Influence of music therapy on the state of anxiety during radiotherapy. Turk J Oncol. 2017;32(4):141-7. doi: http://dx.doi.org/10.5505/ tjo.2017.1689

31. Smith M, Casey L, Johnson D, et al. Music as a therapeutic intervention for anxiety in patients receiving radiation therapy. Oncol Nurs Forum [Internet]. 2001 [cited 2019 Oct 25];28(5):855-62. Available from: http://pesquisa. bvsalud.org/portal/resource/pt/mdl-11421145

32. Kwekkeboom KL. Music versus distraction for procedural pain and anxiety in patients with cancer. Oncol Nurs Forum. 2003;30(3):433-40. doi: http://dx.doi. org/10.1188/03.ONF.433-440

33. Pinto Junior FEL, Ferraz DLM, Cunha EQ, et al. Influência da música na dor e na ansiedade decorrentes de cirurgia em pacientes com câncer de mama. Rev Bras Cancerol. 2012;58(2):135-41. doi: https://doi. org/10.32635/2176-9745.RBC.2012v58n2.611 
34. O'Callaghan C, Sproston M, Wilkinson K, et al. Effect of self-selected music on adults' anxiety and subjective experiences during initial radiotherapy treatment: a randomised controlled trial and qualitative research. J Med Imaging Radiat Oncol. 2012 Aug;56(4):473-7. doi: https://doi.org/10.1111/j.1754-9485.2012.02395.x

35. Yates GJ, Silverman MJ. Immediate effects of singlesession music therapy on affective state in patients on a post-surgical oncology unit: a randomized effectiveness study. Arts Psychother. 2015;44:57-61. doi: https://doi. org/10.1016/j.aip.2014.11.002

36. Bulfone T, Quattrin R, Zanotti R, et al. Effectiveness of music therapy for anxiety reduction in women with breast cancer in chemotherapy treatment. Holist Nurs Pract. 2009;23(4):238-42. doi: http://dx.doi.org/10.1097/ HNP.0b013e3181aeceee

37. Mische Lawson L, Glennon C, Fiscus V, et al. Effects of making art and listening to music on symptoms related to blood and marrow transplantation. Oncol Nurs Forum. 2016 Mar;43(2):E56-63. doi: http://dx.doi. org/10.1188/16.ONF.E56-E63

38. Jasemi M, Aazami S, Zabihi RE. The effects of music therapy on anxiety and depression of cancer patients. Indian J Palliat Care. 2016;22(4):455-8. doi: http:// dx.doi.org/10.4103/0973-1075.191823

39. Goodwin LK. The efficacy of guided imagery to enhance approach coping, emotional expressiveness, and psychological well-being of women with breast cancer [dissertation on the Internet]. [Florida]: University of Florida; 2004 [cited 2019 Oct 25]. 141p. Available from: https:/ufdc.ufl.edu/AA00013623/00001/1x

40. Liu X, Yang H, Zou R, et al. The effect of music therapy and countermeasures design during cancer therapy in China. Psycho-oncology. 2014;23(Supppl 3):193. doi: https://doi.org/10.1111/j.1099-1611.2014.3695

41. Wang Y, Tang H, Guo Q, et al. Effects of intravenous patient-controlled sufentanil analgesia and music therapy on pain and hemodynamics after surgery for lung cancer: a randomized parallel study. J Altern Complement Med. 2015 Nov;21(11):667-72. doi: https://doi.org/10.1089/ acm.2014.0310

42. Chi GC-H-L, Young A, McFarlane J, et al. Effects of music relaxation video on pain and anxiety for women with gynaecological cancer receiving intracavitary brachytherapy: a randomised controlled trial. J Res Nurs. 2015 Mar;20(2):129-44. doi: https://doi. org/10.1177/1744987114529298

43. Bradt J, Potvin N, Kesslick A, et al. The impact of music therapy versus music medicine on psychological outcomes and pain in cancer patients: a mixed methods study. Support Care Cancer. 2015 May;23(5):1261-71. doi: https://doi.org/10.1007/s00520-014-2478-7

44. Liao J, Wu Y, Zhao Y, et al. Progressive muscle relaxation combined with Chinese medicine five-element music on depression for cancer patients: a randomized controlled trial. Chin J Integr Med. 2018 May;24(5):343-7. doi: https://doi.org/10.1007/s1 1655-017-2956-0

45. Burns DS, Meadows AN, Althouse S, et al. Differences between supportive music and imagery and music listening during outpatient chemotherapy and potential moderators of treatment effects. J Music Ther. 2018 Mar;55(1):83-108. doi: https://doi.org/10.1093/jmt/ thy001

46. Sabo CE, Michael SR. The influence of personal message with music on anxiety and side effects associated with chemotherapy. Cancer Nurs. 1996 Aug;19(4):283-9. doi: https://doi.org/10.1097/00002820-199608000-00004

47. Romito F, Lagattolla F, Costanzo C, et al. Music therapy and emotional expression during chemotherapy. How do breast cancer patients feel ? Eur J Integr Med. 2013;5(5):438-442. doi: https://doi.org/10.1016/j. eujim.2013.04.001

48. Zhou K, Li X, Li J, et al. A clinical randomized controlled trial of music therapy and progressive muscle relaxation training in female breast cancer patients after radical mastectomy: results on depression, anxiety and length of hospital stay. Eur J Oncol Nurs. 2015 Feb;19(1):54-9. doi: https://doi.org/10.1016/j.ejon.2014.07.010

49. Ferrer AJ. The effect of live music on decreasing anxiety in patients undergoing chemotherapy treatment. J Music Ther. 2007;44(3):242-55. doi: https://doi.org/10.1093/ jmt/44.3.242

50. Alam M, Roongpisuthipong W, Kim NA, et al. Utility of recorded guided imagery and relaxing music in reducing patient pain and anxiety, and surgeon anxiety, during cutaneous surgical procedures: a single-blinded randomized controlled trial. J Am Acad Dermatol. 2016;75(3):585-9. doi: http://dx.doi.org/10.1016/j. jaad.2016.02.1143

51. Chen SC, Chou CC, Chang HJ, et al. Comparison of group vs self-directed music interventions to reduce chemotherapy-related distress and cognitive appraisal: an exploratory study. Support Care Cancer. 2018;26(2):461-9. doi: http://dx.doi.org/10.1007/ s00520-017-3850-1

52. Horne-Thompson A, Grocke D. The effect of music therapy on anxiety in patients who are terminally ill. J Palliat Med. 2008;11(4):582-90. doi: https://doi. org/10.1089/jpm.2007.0193

53. Eckhouse DR, Hurd M, Cotter-Schaufele S, et al. A randomized controlled trial to determine the effects of music and relaxation interventions on perceived anxiety in hospitalized patients receiving orthopaedic or cancer treatment. Orthop Nurs. 2014;33(6):342-51. doi: https://doi.org/10.1097/ NOR.0000000000000098

54. Lin MF, Hsieh YJ, Hsu YY, et al. A randomised controlled trial of the effect of music therapy and verbal relaxation 
on chemotherapy-induced anxiety. J Clin Nurs. 2011 Apr;20(7-8):988-99. doi: https://doi.org/10.1111/ j.1365-2702.2010.03525.x

55. Li XM, Zhou KN, Yan H, et al. Effects of music therapy on anxiety of patients with breast cancer after radical mastectomy: a randomized clinical trial. J Adv Nurs. 2011 Oct;68(5):1145-55. doi: http://dx.doi. org/10.1111/j.1365-2648.2011.05824.x

56. Dóro CA, Neto JZ, Cunha R, et al. Music therapy improves the mood of patients undergoing hematopoietic stem cells transplantation (controlled randomized study). Support Care Cancer. 2016 Dec;25(3):1013-8. doi: https://doi.org/10.1007/s00520-016-3529-z

57. Rosen J, Lawrence R, Bouchard M, et al. Massage for perioperative pain and anxiety in placement of vascular access devices. Adv Mind Body Med. 2013;27(1):12-23.

58. Maher CG, Sherrington C, Herbert RD, et al. Reliability of the PEDro scale for rating quality of randomized controlled trials. Phys Ther. 2003;83(8):713-21. doi: https://doi.org/10.1093/ptj/83.8.713

59. Bilgiç Ş, Acaroğlu R. Effects of listening to music on the comfort of chemotherapy patients. West J Nurs Res. 2017;39(6):745-62. doi: https://doi. org $/ 10.1177 / 0193945916660527$

60. Imran S, Moosabba MS, Ancheril A. Effects of music therapy on anxiety, blood pressure and respiratory rate in patients undergoing chemotherapy. Nurs Care Open Access J. 2017;2(6):156-8. doi: https://doi. org/10.15406/ncoaj.2017.02.00053

61. Wu PY, Huang ML, Lee WP, et al. Effects of music listening on anxiety and physiological responses in patients undergoing awake craniotomy. Complement Ther Med. 2017;32:56-60. doi: http://dx.doi.org/10.1016/j. ctim.2017.03.007

62. Silva AV, Zandonade E, Amorim MHC. Anxiety and coping in women with breast cancer in chemotherapy. Rev Lat Am Enfermagem. 2017;25:e2891. doi: http:// dx.doi.org/10.1590/1518-8345.1722.2891

63. Menezes NNT, Schulz VL, Peres RS. Impacto psicológico do diagnóstico do câncer de mama: um estudo a partir dos relatos de pacientes em um grupo de apoio. Estud Psicol. 2012;17(2):233-40. doi: http://dx.doi. org/10.1590/S1413-294X2012000200006

64. Saço LF, Da Cunha CFB, Silva RA, et al. Ansiedade em mulheres com câncer de mama e sua relação com a atividade física. HU Revista [Internet]. 2012 [acesso 2019 out. 25];38(3-4):187-92. Disponível em: https://periodicos.uff.br/index.php/hurevista/article/ view/2050/743

65. Fernandes AFC, Bonfim IM, Araújo IMA, et al. Significado do cuidado familiar à mulher mastectomizada. Esc Anna Nery. 2012;16(1):27-33. doi: http://dx.doi. org/10.1590/S1414-81452012000100004

66. Ferreira AS, Bicalho BP, Oda JMM, et al. Câncer de mama: estimativa da prevalência de ansiedade e depressão em pacientes em tratamento ambulatorial. Arq Cienc e Saúde UNIPAR. 2015;19(3):185-9.

67. Silva LC. Câncer de mama e sofrimento psicológico: aspectos relacionados ao feminino. Psicol Estud. 2008;13(2):231-7. doi: http://dx.doi.org/10.1590/ S1413-73722008000200005 